\title{
COMMENT
}

\section{Searching for treatments for non-G12C-KRAS mutant cancers}

\author{
Christina Guo ${ }^{1,2}$ and Udai Banerji (iD ${ }^{1,2}$
}

KRAS mutations drive a wide variety of cancers. Drugs targeting the protein product of $K R A S^{\mathrm{G} 12 \mathrm{C}}$ mutations are currently being evaluated show preliminary efficacy in clinical trials. A clinical trial of VS-6766, a dual RAF-MEK inhibitor, has reported early single agent activity in non-G12C mutated KRAS driven cancers.

British Journal of Cancer (2021) 125:625-626; https://doi.org/10.1038/s41416-021-01357-2

\section{MAIN}

KRAS mutations are commonly associated with a range of solid tumours and haematological malignancies. ${ }^{1}$ Initial attempts to drug KRAS proved challenging due to the high affinity of KRAS to GTP and the relative abundance of GDP/GTP in human cellular tissue. ${ }^{2}$ This led a plethora of efforts to target downstream effectors such as RAF, MEK, PI3K, AKT, mTOR and the combination of these signalling nodes, ${ }^{1-3}$ but these approaches have not led to registration of a drug or drug combinations in the setting of KRAS mutant $\left(K R A S^{\mathrm{M}}\right)$ cancers. There has however been significant progress in developing drugs directly targeting protein products of specific subsets of KRAS mutations i.e. KRAS ${ }^{\mathrm{G} 12 \mathrm{C}}$. These inhibitors (sotorasib and adagrasib) have shown promising activity in non-small cell lung cancer (NSCLC) with KRAS ${ }^{\mathrm{G} 12 \mathrm{C}}$ mutations $\mathrm{s}^{4,5}$ and will no doubt be further explored in registration enabling studies.

Guo et al. reported a Phase $1 \mathrm{~b}$ dose-escalation, basket expansion study of a dual RAF-MEK inhibitor, VS-6766 (previously known as $\mathrm{CH} 5126766$ and RO5126766), which showed promising anti-tumour activity in patients with solid tumours and multiple myeloma harbouring non-G12C-KRAS mutations. ${ }^{6}$ There are several interesting aspects to this study.

Firstly, the drug has interesting pharmacological properties. It is a MEK inhibitor but also blocks in-complex (CRAF-MEK) phosphorylation of MEK, leading to reduction of phosphorylation of both MEK and ERK as demonstrated in pre- and post-treatment biopsies. This is distinct to a reduction of phosphorylation of ERK but not MEK caused by currently licensed MEK inhibitors. The inhibition of signalling in two distinct nodes in the MEK-ERK signalling network by a single drug may explain the preliminary single agent efficacy of VS-6766 reported.

Secondly, the authors have exploited an unusually long halflife of the drug of approximately 50 hours (which is significantly longer than other RAF or MEK inhibitors) to run pharmacokinetic simulations to predict drug concentrations and design a twice a week dosing schedule. The highly intermittent twice a week schedule established in the trial enables patients to tread the fine line between efficacy and toxicity. Further, a dose modification strategy to drop to three-weeks on-one week off rather than reducing the dose of the drug is different from dose modification strategies commonly used in combination studies of targeted agents. ${ }^{8}$ This reflects previous preclinical studies by the group where they had studied the importance of maximal inhibition of MEK signalling in $K R A S^{\mathrm{M}}$ models. ${ }^{9}$ The intermittent schedule has significantly improved the therapeutic index of the drug compared with previous studies that explored continuous dosing schedules. ${ }^{10}$

Finally, the authors have demonstrated single agent activity in a variety of $K R A S^{\mathrm{M}}$ cancers in a cohort of heavily pre-treated patients. Of note, they have demonstrated partial responses in 3/ 10 patients with $K R A S^{\mathrm{M}}$ NSCLC as a single agent and interestingly two of the three patient who responded had KRAS $S^{\mathrm{G} 12 \mathrm{~V}}$ mutations. Given there are no KRAS targeted therapies for KRAS ${ }^{\mathrm{G} 12 \mathrm{~V}}$ mutations, VS-6766 could be explored further in this disease space both as a single agent or in combination. Of note, 3/5 patients with $R A S / R A F$ mutations with gynaecological cancers responded to treatment. There is considerable excitement and activity of MEK inhibitors (trametinib and binimetinib) in low grade serous ovarian cancer (LGSOC). ${ }^{11,12}$ The combination study of VS-6766 with defactinib have shown promising activity in Phase 1 studies with expansions in $\mathrm{LGSOC}^{13}$ and randomised a Phase 2 trial is exploring the activity of the combination in LGSOC is ongoing (NCT04625270). Further, an interesting response was seen in patient with $K R A S^{\mathrm{G} 12 \mathrm{~V}}$ driven multiple myeloma. While anecdotal responses of MEK inhibitors have been noted in myeloma, ${ }^{14}$ this further confirms response of this agent in an independent cancer type driven by KRAS mutations.

Attention to detail of pharmacokinetics, pharmacodynamics, toxicity and predictive biomarkers of efficacy are part of the pharmacological audit trail and remain critical to successful development of targeted therapy ${ }^{15}$ (Fig. 1). The trial by Guo et al. ${ }^{6}$ has defined a backbone of a highly intermittent schedule of novel RAF-MEK inhibitor VS-6766. Multiple early Phase clinical trials of combinations with this VS-6766 are currently ongoing with agents such as everolimus (NCT02407509) and defactinib (NCT03875820). Randomised studies of the combination of VS-6766 and defactinib to explore efficacy of the combination in KRAS ${ }^{M}$ LGSOC (NCT04625270) and NSCLC (NCT04620330) are currently ongoing.

\footnotetext{
${ }^{1}$ The Institute of Cancer Research, London, UK and ${ }^{2}$ The Royal Marsden Hospital NHS Foundation Trust, London, UK
} Correspondence: Udai Banerji (udai.banerji@icr.ac.uk) 


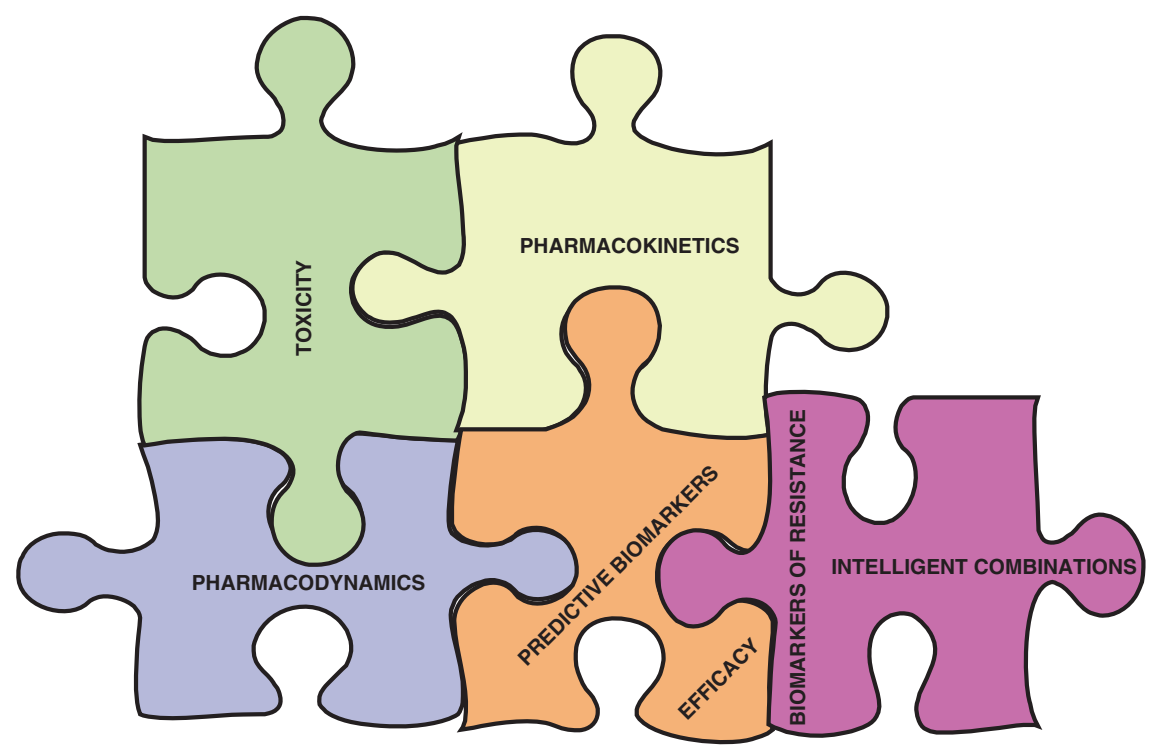

Fig. 1 The Pharmacological Audit Trial. Crucial elements in the pharmacological audit trail that are key to optimise dosing schedules and use predictive biomarkers to accelerate early clinical development.

\section{AUTHOR CONTRIBUTIONS}

C.G. and U.B. contributed to the writing of the manuscript.

\section{ADDITIONAL INFORMATION}

Ethics approval and consent to participate Not applicable.

Data availability Not applicable.

Competing interests U.B. has received funding for the investigator-initiated clinical trial discussed in the manuscript from Chugai Pharmaceuticals and Verastem Oncology. U.B. has also received funding from Verastem Oncology for preclinical research. U.B. is an employee of The Institute of Cancer Research that has commercial interests in the development of RAF inhibitors.

Funding information The authors acknowledge funding from the Experimental Cancer Medicine Centre and Biomedical Research Centre to the Institute of Cancer Research and the Royal Marsden Hospital NHS foundation trust. U.B. also acknowledges funding from CRUK and the NIHR.

Publisher's note Springer Nature remains neutral with regard to jurisdictional claims in published maps and institutional affiliations.

\section{REFERENCES}

1. Uprety, D. \& Adjei, A. A. KRAS: from undruggable to a druggable cancer target. Cancer Treat. Rev. 89, 102070 (2020).

2. Cox, A. D., Fesik, S. W., Kimmelman, A. C., Luo, J. \& Der, C. J. Drugging the undruggable RAS: mission possible? Nat. Rev. Drug Discov. 13, 828-851 (2014).

3. Hymowitz, S. G. \& Malek, S. Targeting the MAPK pathway in RAS mutant cancers. Cold Spring Harb. Perspect. Med. 8, a031492 (2018).

4. Hong, D. S., Fakih, M. G., Strickler, J. H., Desai, J., Durm, G. A., Shapiro, G. I. et al. KRAS(G12C) inhibition with sotorasib in advanced solid tumors. N. Engl. J. Med. 383, 1207-1217 (2020).

5. Janne, P., Rybin, I., Sipra, A., Riely, G., Papadopoulous, K., Sabari J. et al. KRYSTAL-1: activity and safety of Adagrasib (MRTX849) in advanced/metastatic non-smallcell lung cancer (NSCLC) harboring KRAS G12C mutation. Eur. J. Cancer 138, S1-S2 (2020).

6. Guo, C., Chenard-Poirier, M., Roda, D., de Miguel, M., Harris, S. J., Candilejo, I. M. et al. Intermittent schedules of the oral RAF-MEK inhibitor CH5126766/VS-6766 in patients with RAS/RAF-mutant solid tumours and multiple myeloma: a singlecentre, open-label, phase 1 dose-escalation and basket dose-expansion study. Lancet Oncol. 21, 1478-1488 (2020).
7. Lito, P., Saborowski, A., Yue, J., Solomon, M., Joseph, E., Gadal, S. et al. Disruption of CRAF-mediated MEK activation is required for effective MEK inhibition in KRAS mutant tumors. Cancer Cell 25, 697-710 (2014).

8. Lopez, J. S. \& Banerji, U. Combine and conquer: challenges for targeted therapy combinations in early phase trials. Nat. Rev. Clin. Oncol. 14, 57-66 (2017).

9. Stewart, A., Thavasu, P., de Bono, J. S. \& Banerji, U. Titration of signalling output: insights into clinical combinations of MEK and AKT inhibitors. Ann. Oncol. 26, 1504-1510 (2015)

10. Martinez-Garcia, M., Banerji, U., Albanell, J., Bahleda, R., Dolly, S., Kraeber-Bodere, F. et al. First-in-human, phase I dose-escalation study of the safety, pharmacokinetics, and pharmacodynamics of RO5126766, a first-in-class dual MEK/ RAF inhibitor in patients with solid tumors. Clin. Cancer Res. 18, 4806-4819 (2012).

11. Gershenson, D., Brady, W., Paul, J., Carty, K., Rodgers, W., Millian, D. et al. A randomized phase II/III study to assess the efficacy of trametinib in patients with recurrent or progressive low-grade serous ovarian or peritoneal cancer. Ann. Oncol. 30, v897-v898 (2019).

12. Monk, B. J., Grisham, R. N., Banerjee, S., Kalbacher, E., Mirza, M. R., Romero, I. et al. MILO/ENGOT-ov11: binimetinib versus physician's choice chemotherapy in recurrent or persistent low-grade serous carcinomas of the ovary, fallopian tube, or primary peritoneum. J. Clin. Oncol. 38, 3753-3762 (2020).

13. Shinde, R., Terbuch, A., Little, M., Caldwell, R., Kurup, R., Riisnaes, R. et al. Phase I study of the combination of a RAF-MEK inhibitor CH5126766 and FAK inhibitor defactinib in an intermittent dosing schedule with expansions in KRAS mutant cancers. Cancer Res. 80(16 Suppl), Abstract CT143 (2020).

14. Heuck, C. J., Jethava, Y., Khan, R., van Rhee, F., Zangari, M., Chavan, S. et al. Inhibiting MEK in MAPK pathway-activated myeloma. Leukemia 30, 976-980 (2016).

15. Banerji, U. \& Workman, P. Critical parameters in targeted drug development: the pharmacological audit trail. Semin. Oncol. 43, 436-445 (2016).

Open Access This article is licensed under a Creative Commons Attribution 4.0 International License, which permits use, sharing, adaptation, distribution and reproduction in any medium or format, as long as you give appropriate credit to the original author(s) and the source, provide a link to the Creative Commons license, and indicate if changes were made. The images or other third party material in this article are included in the article's Creative Commons license, unless indicated otherwise in a credit line to the material. If material is not included in the article's Creative Commons license and your intended use is not permitted by statutory regulation or exceeds the permitted use, you will need to obtain permission directly from the copyright holder. To view a copy of this license, visit http://creativecommons. org/licenses/by/4.0/.

(c) The Author(s) 2021 